Copyright (C 2016 IEEE. Personal use of this material is permitted. Permission from IEEE must be obtained for all other uses, in any current or future media, including reprinting/republishing this material for advertising or promotional purposes, creating new collective works, for resale or redistribution to servers or lists, or reuse of any copyrighted component of this work in other works. 


\title{
A Novel Method of Measuring Transformer Oil Interfacial Tension using UV-Vis Spectroscopy
}

\author{
Norazhar Abu Bakar ${ }^{1,2}$ and A. Abu-Siada ${ }^{1}$ \\ ${ }^{1}$ Electrical and Computer Engineering Department, Curtin University, Western Australia \\ ${ }^{2}$ Faculty of Electrical Engineering, Universiti Teknikal Malavsia Melaka, Malavsia
}

\section{SUMMARY}

A new method of estimating the interfacial tension of transformer oil, using ultraviolet-to-visible spectroscopy and two artificial intellegence models, is proposed.

Key Words: Interfacial tension, Transformer insulation oil, UVVis spectroscopy, Artificial neural network, Fuzzy logic.

\section{INTRODUCTION}

$\mathrm{R}$ eliable monitoring and diagnostic techniques for detecting transformer incipient faults are required in order to help avoiding catastrophic failures, and to inform an efficient predictive maintenance program which improves the reliability of the equipment $[1,2]$. The overall health of an operating transformer depends largely on the condition of its insulation system [3]. Long term degradation of an insulation system occurs mainly through heating (pyrolysis), moisture ingress (hydrolysis) and air ingress (oxidation) [4, 5]. Incipient faults within a transformer can be detected by analyzing samples of its insulating oil, e.g., using dissolved gas analysis and furan analysis [6]-[8]. Interfacial tension (IFT) and acid number measurements may also be used to examine the oil quality [9]. Although polar compounds in transformer oil usually have little or no acidity, they can react to form acids which increase the rate of insulation paper ageing. IFT and acid number measurements can therefore provide early warning of such ageing [10].

The ASTM D971 standard (Interfacial Tension of Oil against Water by the Ring Method) is widely used to measure IFT of insulating oil [11], [12]. Soluble polar contaminants and degradation products affect the physical and electrical properties of the insulating oil [11], [12], thereby lowering the IFT. According to the ASTM D971 standard, a planar ring of platinum wire with circumference $d$ is immersed in a container filled with water and insulating oil. The force $\mathrm{F}$ required to move the ring ten millimetres upwards through the oil-water interface is measured, and the IFT of the oil is then given by F/d [12]. The IFT of new insulating oil is expected to be in the range $40-50 \mathrm{mN} / \mathrm{m}$; an oil sample with an IFT value less than $25 \mathrm{mN} / \mathrm{m}$ is considered to be approaching the end of its useful service lifetime [13].

The IFT measurement technique requires great care. Wrong or inconsistent data are likely to be obtained if the precautions mentioned in [12] are not carefully and completely observed. A trained person is required to make the measurements, using an expensive piece of equipment, so that in nearly all cases the oil samples have to be sent to an external laboratory.

In this article we propose an alternative method of measuring the IFT of transformer oil, using ultraviolet-tovisible (UV-Vis) spectroscopy and artificial intelligence (AI). The UV-Vis spectrum of the oil can be measured very quickly onsite, using equipment which is much less costly than that described in [12], and special training is not required to make the measurements, which can be automated if required. An artificial neural network (ANN) and a fuzzy logic model have been developed to analyze the UV-Vis spectral data. The IFT values are compared with those obtained using the ASTM D971 standard method. The accuracy of the two AI models is tested using another set of oil samples with a wide range of IFT values.

\section{Proposed UV-VIS Spectroscopy Method}

Spectroscopic measurements play an important part in various power transformer condition monitoring applications [6], [14]-[17]. They are also widely used to determine energy levels in atoms and molecules [18]-[20]. We made IFT measurements on several samples of transformer oil, using UV-Vis spectroscopy in accordance with ASTM E275 [21]. Our UV-Vis spectroscopy experimental setup is shown in Figure 1.

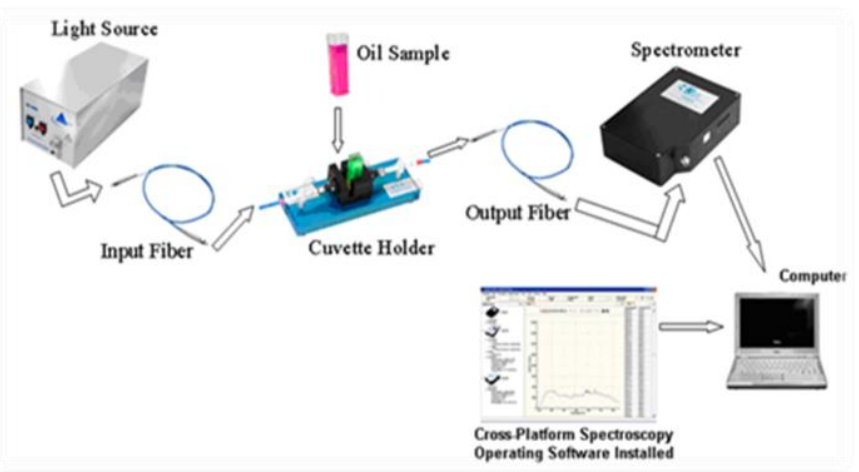

Figure 1. Experimental setup for UV-Vis spectroscopy

A light beam (wavelength $\lambda$ in the range 200-1100 nm) travels through an optical fiber and enters the oil sample, which is placed in a cuvette. The light interacts with the oil, and the transmitted light passes through a second optical fiber and enters the spectrometer, which is connected to a computer which displays and analyzes the spectral response of the oil sample. The light absorbance $A_{\lambda}$ of the oil sample at wavelength $\lambda$ is related to the ratio of the light intensity transmitted through the oil sample to the light intensity incident on the sample. According to the Beer-Lambert Law [19]

$$
A_{\lambda}=-\log _{10}\left(\frac{S_{\lambda}-D_{\lambda}}{R_{\lambda}-D_{\lambda}}\right)=\varepsilon_{\lambda} . c .1
$$

where $S_{\lambda}$ is the light intensity transmitted through the sample, $R_{\lambda}$ is the light intensity incident on the sample, $D_{\lambda}$ is the 
intensity detected by the spectrometer with no light incident on the sample, $\varepsilon_{\lambda}$ is the absorbance coefficient of the absorbing species (or molecule) in the oil, $c$ is the concentration of the absorbing species in the oil (gram/litre), and $l$ is the path length of the light through the oil.

\section{EXPERIMENTAL}

IFT measurements were made, following the ASTM D971 ring method, on fifty-five mineral oil samples collected from in-service transformers of various ratings, operating under various conditions and with various times in service. All samples were Shell Diala BX containing an additive to retard oil oxidation and delay the formation of acids and sludge [22]. Absorption spectroscopy measurements were then made at $20^{\circ} \mathrm{C}$ on the same samples, using a laboratory grade spectrophotometer in accordance with ASTM E275 [21], [23]. To evaluate the impact of the cuvettes containing the oil samples on the measurements, an empty cuvette was scanned and its spectrum stored as a reference. Approximately $2.0 \mathrm{ml}$ of each oil sample was then placed in a cuvette and scanned. The difference between this spectrum and the reference spectrum yields the true oil absorbance characteristics at various wavelengths from $200 \mathrm{~nm}$ to $1100 \mathrm{~nm}$. In order to avoid contamination of the oil, all samples were handled and stored in accordance with ASTM D923 [24] and measured within 1 to 2 days after extraction from the transformer. Samples of oil absorbance spectra are shown in Figure 2, where the encircled number is the IFT value of the sample in $\mathrm{mN} / \mathrm{m}$. It will be seen that, as the IFT value falls, the peak absorbance rises, and the increase in absorbance is maintained across the "flat top" of each plot. A new oil sample with an IFT of $40 \mathrm{mN} / \mathrm{m}$ exhibits the lowest peak absorbance and the narrowest bandwidth.

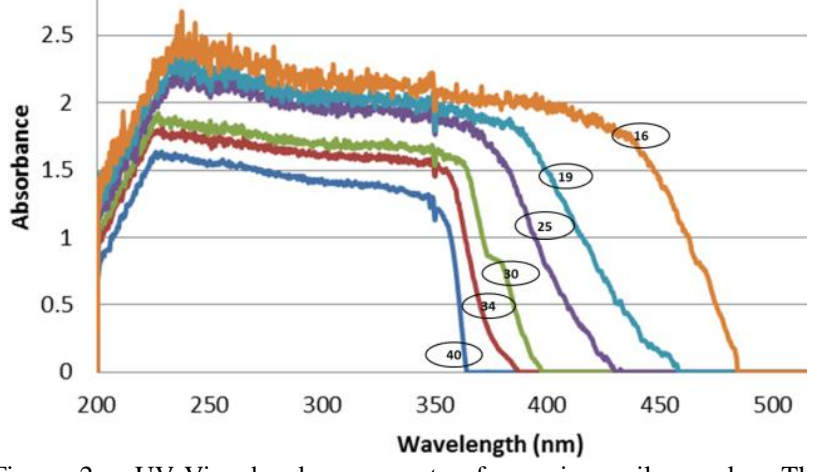

Figure 2. UV-Vis absorbance spectra for various oil samples. The encircled numbers are the corresponding IFT values (in $\mathrm{mN} / \mathrm{m}$ ) [28].

The impact of acidity [27] on spectral response was also examined. IFT and acid number are shown in the 3-D plots of Figure 3 as functions of peak absorbance and the longest wavelength at which absorbance could be detected. There is no consistent correlation between acid number and either of these two quantities. However, as shown in Figure 3, the peak absorbance and the longest wavelength at which absorbance could be detected increased with decreasing IFT [28].
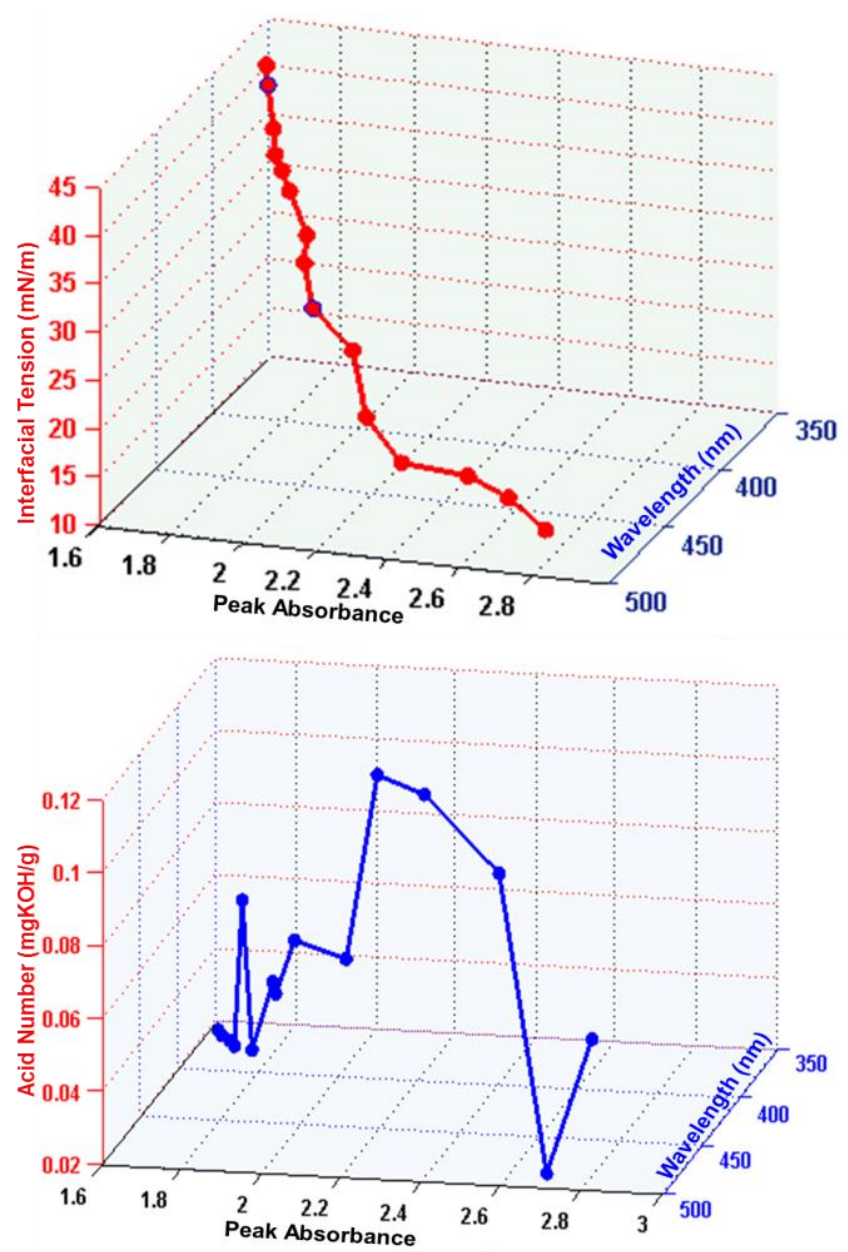

Figure 3. IFT and acid number as functions of peak absorbance and the longest wavelength at which absorbance could be detected.

\section{ARTIFICIAL INTELLIGENCE MODELS}

An artificial neural network model and a fuzzy logic model have been developed to investigate the correlation between the IFT of an oil sample and its spectral response parameters (bandwidth and peak absorbance).

\section{A. Artificial Neural Network Model}

An artificial neural network (ANN) is a machine learning technique modelled on biological neurons. Its weighted inputs determine the activation of the artificial neuron, using a mathematical function [29]. The output of the neuron is determined by another mathematical function, and depends on certain pre-determined threshold limits. Since an ANN is "self-taught", and has the ability to deal with complex situations, it has been implemented for various applications, e.g., predicting the furan content of transformer oil [30], transformer failure diagnosis [31] and analysis of gases dissolved in transformer oil [32].

Basic ANN architecture consists of an input layer, a hidden layer, and an output layer. In this work the input layer consisted of two components, namely the bandwidth and peak absorbance of the transformer oil, the hidden layer consisted of 20 neurons, and the output was the predicted IFT value. 


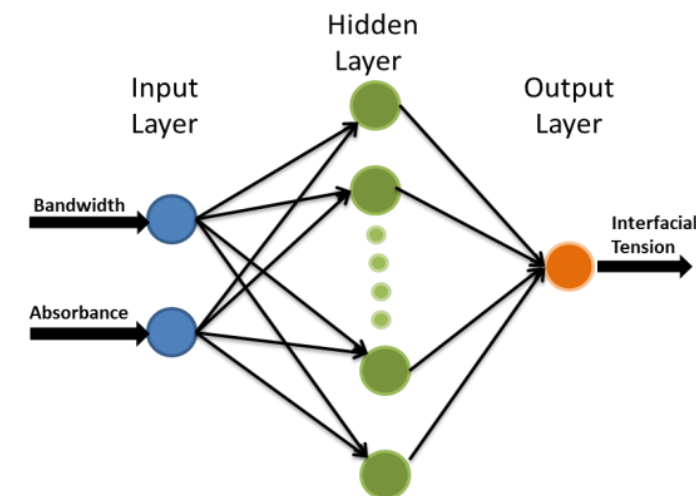

Figure 4. Architecture of the artificial neural network model developed in this work.

The network was trained using the Levenberg-Marquardt back propagation algorithm [29], which adopts supervised learning in order to correlate the independent input variables (peak absorbance and bandwidth of the absorption spectrum) and the dependent output variable (IFT value of the oil). Random weightings of the neurons were carefully adjusted to minimize the difference between the measured and estimated IFT values. Due to the limited amount of input and output data, a cross validation method was used to validate the ANN model [33]; it minimises biasing error between the estimated and measured IFT values, and enhances the correlation coefficient between the input and output parameters.

The oil samples were randomly divided into three sets, 39 for training purposes, 8 for validation of the ANN, and 8 for testing the performance of the ANN. The training set was used to determine the weights of the neurons, the validation set was used to determine the optimum number of neurons in the hidden layer, and the testing set was used to evaluate the overall performance of the ANN model.

\section{B. Fuzzy Logic Model}

Fuzzy logic is frequently used in modelling non-linear or complex systems [34]. It uses variables, usually in the form of words, which are manipulated using logic rules, such as "IFTHEN". Fuzzy logic system architecture consists of five main elements, namely fuzzification, membership function, fuzzy inference engine, fuzzy rules, and defuzzification, as shown in Figure 5. A brief description of these five elements follows:

Membership functions. There are different types of membership function, such as triangular, trapezoidal and Gaussian. As an example, if men's height is divided into three levels, above $170 \mathrm{~cm}$ being Tall, $170-150 \mathrm{~cm}$ being Normal, and less than $150 \mathrm{~cm}$ being Short, then Height is a fuzzy set and Tall, Normal and Short are the membership functions of that fuzzy set.

Fuzzification is the process of allocating the input data among the membership functions. Thus in the above example a man with a height of $140 \mathrm{~cm}$ is allocated to the Short membership function of the Height fuzzy set.

Fuzzy Rules are conditional statements, e.g., "IF-THEN" or "IF-AND-THEN", which associate one or more variables with each other. Thus IF(man is short) AND (man is heavy) THEN (man is overweight).
Inference engine converts the fuzzy inputs to the fuzzy outputs using pre-defined fuzzy rules.

Defuzzification is the process of converting the membership functions of a fuzzy set to an output with a quantitative value. A common defuzzification method is the centre of gravity (COG) method, defined by [34]

$$
Z_{C O G}=\frac{\int z \cdot \mu_{z}(z) d z}{\int \mu_{z}(z) d z}
$$

where $Z_{C O G}$ is the defuzzified output, $\mu_{z}$ is the membership function of the output fuzzy set determined from the input data and the developed fuzzy rules, and $z$ is the output variable of the fuzzy system.

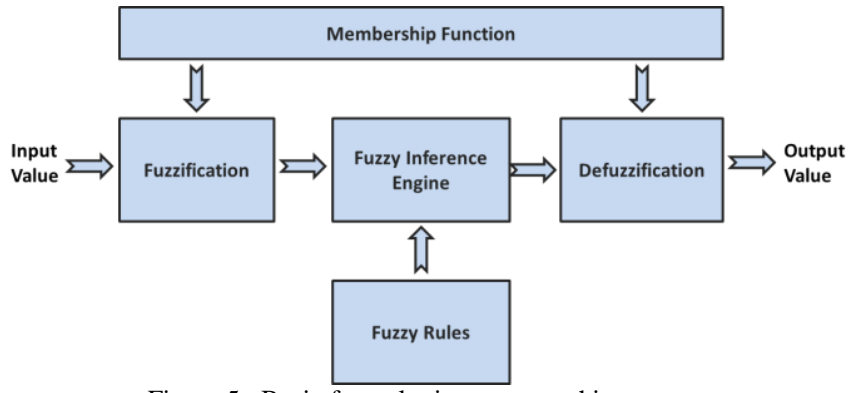

Figure 5. Basic fuzzy logic system architecture.

In this work, the input variables to the fuzzy logic model were the bandwidth (BW) and peak absorbance level (ABS) of the oil sample spectrum, and the output of the model was the estimated IFT of the oil sample. The proposed fuzzy logic model was built using the MATLAB graphical user interface tool box. Each input was fuzzified into five Gaussian membership functions, i.e., low, low-medium, medium, medium-high and high for peak absorbance, and small, smallmedium, medium, medium-high and high for bandwidth, as shown in Figure 6. The IFT output variable was represented by the five triangular membership functions very good, good, marginally critical, bad and very bad. Oil samples with IFTs greater than $40 \mathrm{mN} / \mathrm{m}$ were considered very good, and oil samples with IFTs less than $17 \mathrm{mN} / \mathrm{m}$ were considered very bad.

Twenty-five fuzzy logic (IF-AND-THEN) rules were developed, based on the known correlation between IFT and the spectral parameters BW and ABS, as shown in Figure 3. The fuzzy logic rules are illustrated graphically in Figure 7; the IFT value can be obtained for any pair of peak absorbance and bandwidth values. Samples of these rules are as follows:

- Rule 1: IF (ABS is low) AND (BW is low) THEN (IFT is very good)

- Rule 2: IF (ABS is low) AND (BW is low-medium) THEN (IFT is very good)

- Rule 3: IF (ABS is high) AND (BW is high) THEN (IFT is very bad). 

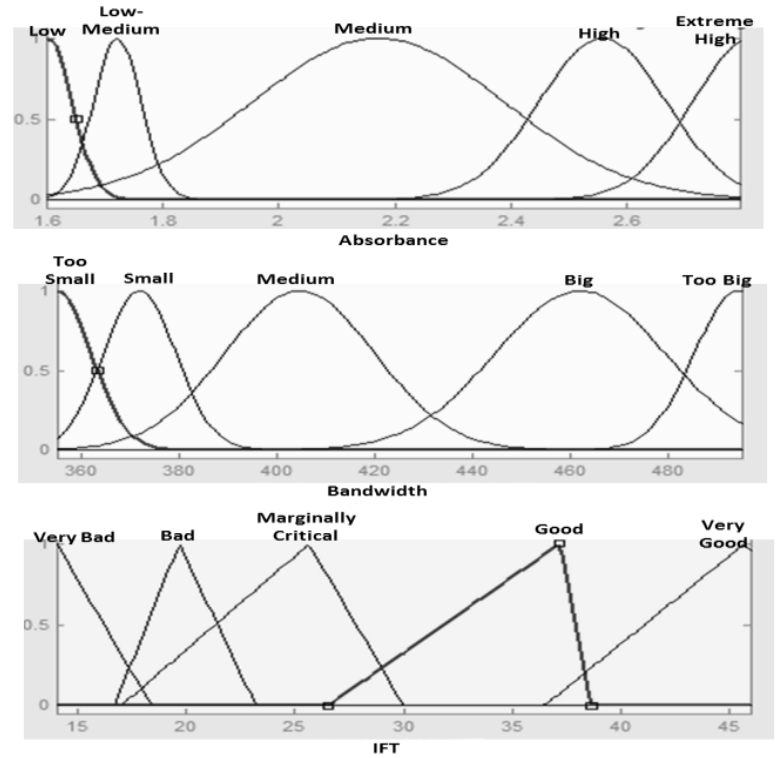

Figure 6. Absorbance, bandwidth and IFT membership functions [35].

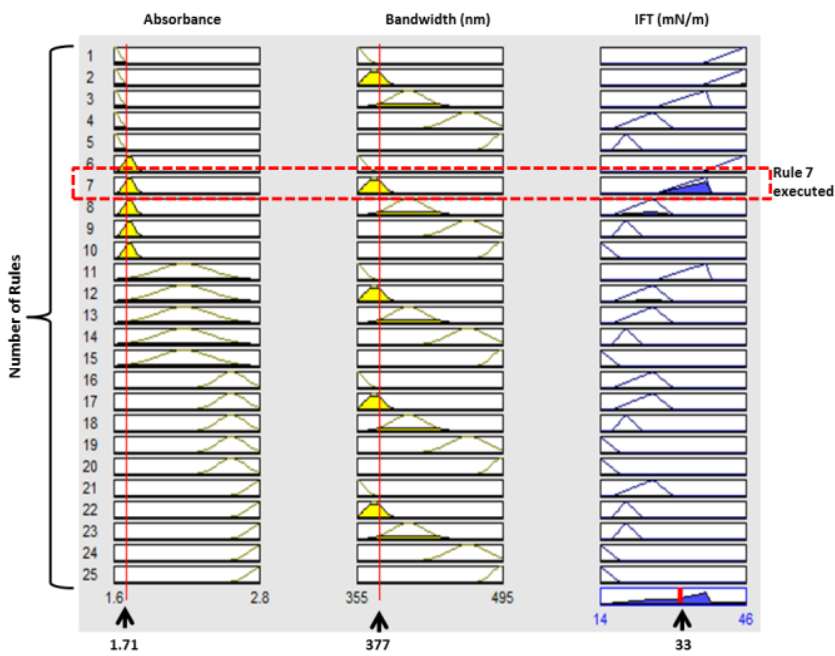

Figure 7. Graphical representation of the developed fuzzy logic rules correlating absorbance and bandwidth with IFT value.

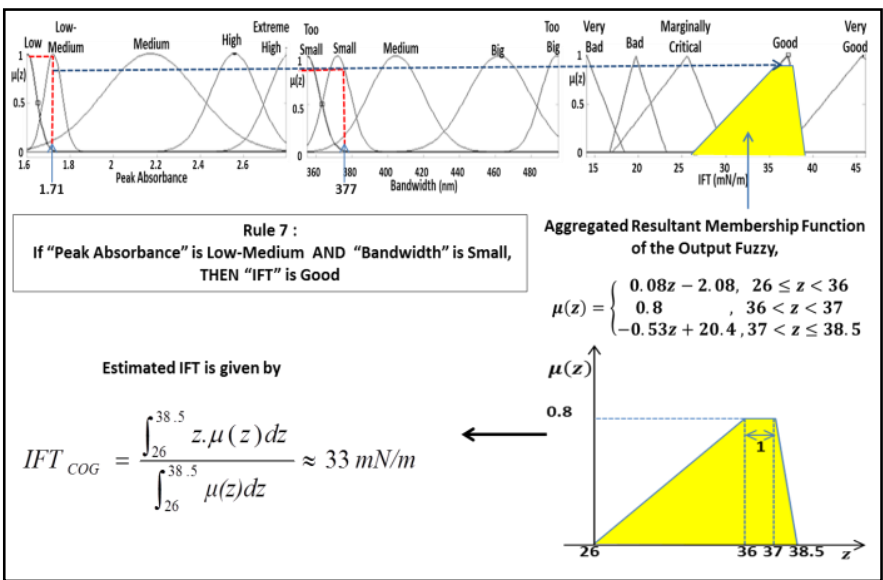

Figure 8. An example of the calculation of IFT based on Figure 7 and (2).

Thus if $\mathrm{ABS}=1.71$ and $\mathrm{BW}=377 \mathrm{~nm}$, then Rule 7 (IF (ABS is low-medium) AND (BW is small-medium) THEN (IFT is good)) is executed, and an estimated IFT of $33 \mathrm{mN} / \mathrm{m}$ is obtained using COG defuzzification, as shown in Figure 8.

\section{RESULTS}

Figures 9 and 10 show the estimated IFT as a function of $\mathrm{ABS}$ and $\mathrm{BW}$, according to the ANN and fuzzy logic models respectively.

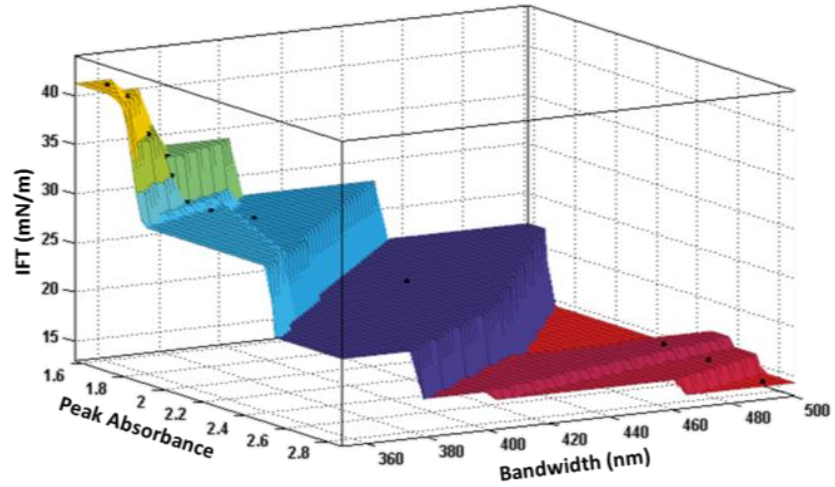

Figure 9. IFT as a function of bandwidth and peak absorbance, according to the ANN model.

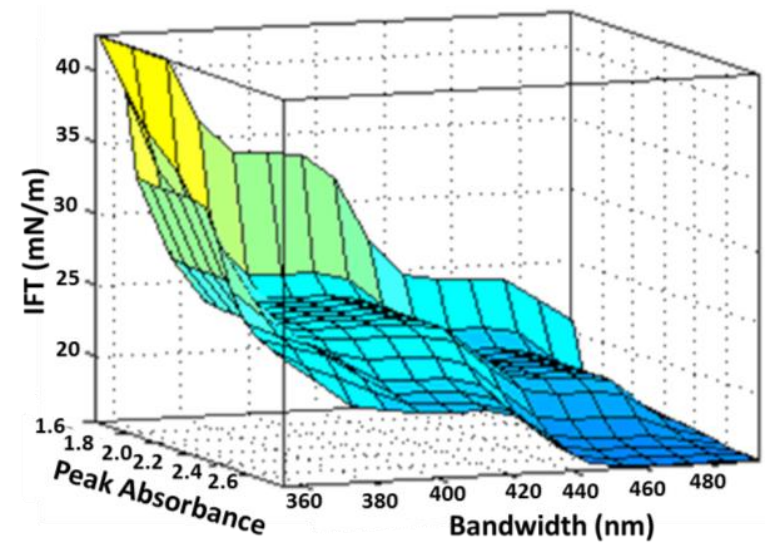

Figure 10. IFT as a function of bandwidth and peak absorbance, according to the fuzzy logic model.

\section{ACCURACY ANALYSIS}

Fifteen oil samples collected from various transformers operating under different conditions were used to examine the accuracy of the proposed models. The IFT of each sample was measured following ASTM D971. The UV-Vis spectral response of each sample was also obtained, and BW and ABS used as input data to the AI estimation models. The resulting IFT values were then compared with the ASTM D971 values (Figure 11).

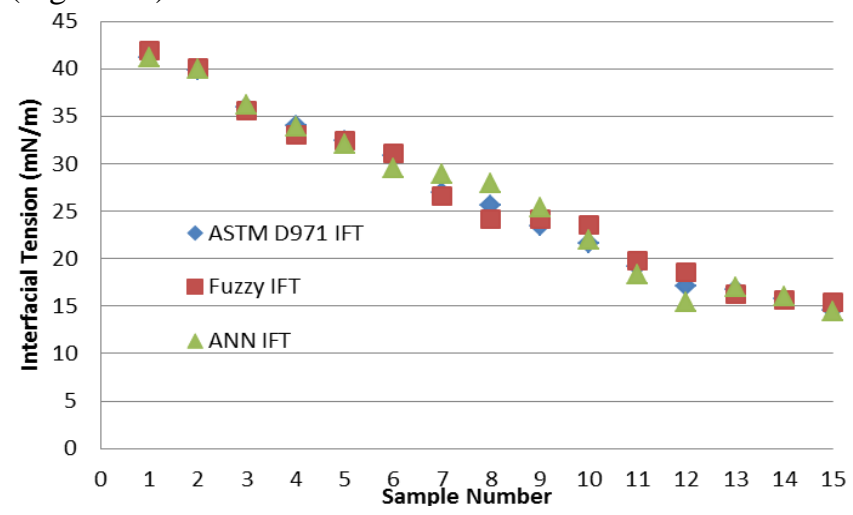

Figure 11. Comparison of ASTM D971, fuzzy logic and ANN values of IFT for each of fifteen oil samples. 
The percentage difference $\mathrm{A}$ between $\mathrm{IFT}_{\mathrm{ASTM}}$ D971 and IFT $_{\text {est }}$ (Fuzzy IFT or ANN IFT) is given by:

$$
A=\left(\frac{I F T_{A S T M D 971}-I F T_{e s t}}{I F T_{\text {ASTMD971 }}}\right) \times 100
$$

where $I F T_{A S T M ~ D 971}$ is the IFT measured using ASTM D971 and $I F T_{\text {est }}$ is the IFT obtained using the ANN or the fuzzy logic model. Averaged over the fifteen samples, A was 3.38\% for the ANN model and $3.25 \%$ for the fuzzy logic model. The overall accuracy, i.e., the ratio of the number of correctly estimated IFT values (where A is less than 5\%) to the total number of tested samples, was $80 \%$ and $87 \%$ for the ANN and fuzzy logic models respectively.

\section{COMPARISON BETWEEN ASTM D971 RING METHOD AND UV-VIS SPECTROSCOPY}

The proposed UV-Vis spectroscopy method and the existing ASTM D971 method are compared in Table I.

Table I

Comparison of the ASTM D971 and the proposed UV-Vis methods of measuring the IFT of oil samples

\begin{tabular}{|c|l|l|}
\hline Method & \multicolumn{1}{|c|}{ ASTM D971 } & \multicolumn{1}{c|}{ UV-Vis } \\
\hline $\begin{array}{c}\text { Advantages } \\
\text { and } \\
\text { Disadvantages }\end{array}$ & $\begin{array}{l}\text { Requires trained person to } \\
\text { prepare the oil sample and } \\
\text { make the measurement. } \\
\text { Must be conducted in a } \\
\text { laboratory environment and } \\
\text { is time consuming. } \\
\text { No possibility of on-line } \\
\text { monitoring. }\end{array}$ & $\begin{array}{l}\text { Does not require a } \\
\text { trained person to make } \\
\text { the measurement. }\end{array}$ \\
\hline Cost & $\begin{array}{l}\text { Can be conducted on- } \\
\text { site. } \\
\text { Could be implemented } \\
\text { online. }\end{array}$ \\
\hline Running Costs $\$ 10,000$ (depending & $\begin{array}{l}\text { US\$120 to US\$150 per } \\
\text { sample. }\end{array}$ & $\begin{array}{l}\text { From US\$3,500 } \\
\text { spectrophotometer } \\
\text { features). }\end{array}$ \\
\hline
\end{tabular}

\section{CONCLUSION}

We have described a novel technique for estimating the IFT of mineral transformer oil samples, using UV-Vis spectroscopy. It is known that there is a strong correlation between the IFT of the oil and two of its spectral response parameters, namely bandwidth and peak absorbance. We have developed two artificial intelligence models to map this correlation. Our technique offers the advantages that it can be used onsite, it does not need specially trained personnel to make the measurements, it has negligible running costs, and can be readily implemented online for continuous monitoring of the condition of the oil.

\section{ACKNOWLEDGMENTS}

The authors thank Min Zaw, Gary Ienco and Mohamed Dihishi, from Transformer Fitness Pty. Ltd., Western Australia, for making available their expert knowledge on transformer oil condition management.

\section{REFERENCES}

[1] A. E. B. Abu-Elanien and M. M. A. Salama, "Survey on the Transformer Condition Monitoring," in Power Engineering, 2007 Large Engineering Systems Conference on, 2007, pp. 187-191.

[2] A. Abu-Siada and S. Islam, "A Novel Online Technique to Detect Power Transformer Winding Faults," IEEE Transactions on Power Delivery, vol. 27, pp. 849-857, Apr 2012.

[3] D. J. Woodcock and J. C. Wright, "Power transformer design enhancements made to increase operational life," in Sixty-Sixth Annual International Conference of Doble Clients, 1999.

[4] M. Arshad and S. M. Islam, "Significance of cellulose power transformer condition assessment," Dielectrics and Electrical Insulation, IEEE Transactions on, vol. 18, pp. 1591-1598, 2011.

[5] A. Schaut, S. Autru, and S. Eeckhoudt, "Applicability of methanol as new marker for paper degradation in power transformers," Dielectrics and Electrical Insulation, IEEE Transactions on, vol. 18, pp. 533-540, 2011.

[6] N. A. Bakar, A. Abu-Siada, and S. Islam, "A review of dissolved gas analysis measurement and interpretation techniques," Electrical Insulation Magazine, IEEE, vol. 30, pp. 39-49, 2014.

[7] N. A. Bakar, A. Abu-Siada, and S. Islam, "A Review on Chemical Diagnosis Techniques for Transformer Paper Insulation Degradation," in Australasian Universities Power Engineering Conference, AUPEC 2013 , 2013.

[8] A. Abu-Siada, S. Hmood, and S. Islam, "A New Fuzzy Logic Approach for Consistent Interpretation of Dissolved Gas-in-Oil Analysis," Dielectrics and Electrical Insulation, IEEE Transactions on, vol. 20, pp. 2343-2349, Dec 2013.

[9] H. Gumilang, "Unique relationship between interfacial tension test (IFT) and neutral number test (Acidity) of transformer insulation oil in PLN P3B JB - Jakarta and Banten Regional," in Properties and Applications of Dielectric Materials, 2009. ICPADM 2009. IEEE 9th International Conference on the, 2009, pp. 29-32.

[10]D. Hydroelectric Research and Technical Services Group, Colorado, "Facilities, Illustrations, Standards and Techniques; Transformer Maintenance," US Department of Interior Bureau of Reclamation, vol. 3 30, pp. 1-81, October 2000.

[11]B. Pahlavanpour, M. Eklund, and K. Sundkvist, "Revised IEC standard for maintenance of in-service insulating oil," in Weidmann Third Annual Technical Conference, Sacramento, USA, 2004.

[12] ASTM, "Standard Test Method for Interfacial Tension of Oil Against Water by the Ring Method," ASTM D971 - 12, 2012.

[13]T. O. Rouse, "Mineral insulating oil in transformers," Electrical Insulation Magazine, IEEE, vol. 14, pp. 6-16, 1998.

[14]A. Abu-Siada, S. P. Lai, and S. M. Islam, "A Novel Fuzzy-Logic Approach for Furan Estimation in Transformer Oil," Power Delivery, IEEE Transactions on, vol. 27, pp. 469-474, 2012.

[15]D. Skelly, "Photo-acoustic spectroscopy for dissolved gas analysis: Benefits and Experience," in Condition Monitoring and Diagnosis (CMD), 2012 International Conference on, 2012, pp. 29-43.

[16]H. Malik, A. K. Yadav, Tarkeshwar, and R. K. Jarial, "Make use of UV/VIS spectrophotometer to determination of dissolved decay products in mineral insulating oils for transformer remnant life estimation with ANN," in India Conference (INDICON), 2011 Annual IEEE, 2011, pp. 16.

[17]H. H. Patrick J. Baird, Gary C. Stevens, "On-Site Analysis of Transformer Paper Insulation Using Portable Spectroscopy for Chemometric Prediction of Aged Condition," Dielectrics and Electrical Insulation, IEEE Transactions on, vol. 15, p. 1089, 2008.

[18]N. A. Bakar, A. Abu-Siada, N. Das, and S. Islam, "Effect of Conducting Materials on UV-Vis Spectral Response Characteristics," Universal Journal of Electrical and Electronic Engineering, pp. 81-86, 2013.

[19]H. Kaur, Spectroscopy. Meerut, IND: Global Media, 2009.

[20]L. D. Field, S. Sternhell, and J. R. Kalman, Organic Structures from Spectra, 4th ed.: Wiley, 2011.

[21] ASTM, "Standard Practice for Describing and Measuring Performance of Ultraviolet and Visible Spectrophotometers," ASTM E275, 2013.

[22] J. Wada, G. Ueta, S. Okabe, and T. Amimoto, "Inhibition technique of transformer insulating oil degradation-evaluation of the effectiveness of oxidation degradation inhibitors," Dielectrics and Electrical Insulation, IEEE Transactions on, vol. 20, pp. 1641-1648, 2013.

[23] ASTM, "Standard Practice for Describing and Measuring Performance of Ultraviolet, Visible, and Near-Infrared Spectrophotometers,", ASTM E275-01, vol. 03.06, pp. 72-81, 2001. 
[24]ASTM, "Standard Practices for Sampling Electrical Insulating Liquids," ASTM D923-07, 2007.

[25]IEC, "Insulating liquids - Determination of the breakdown voltage at power frequency - Test method," IEC 60156 Standard, 1995.

[26]IEC, "Insulating Liquids- Oil-impregnated paper and pressboardDetermination of water by automatic coulometric Karl Fischer titration," IEC 60814 Standard, 1997.

[27]IEC, "Insulating liquids - Determination of acidity - Part 2: Colourimetric titration," IEC 62021-2 Standard, 2007.

[28]N. A. Bakar, A. Abu-Siada, S. Islam, and M. F. El-Naggar, "A New Technique to Measure Interfacial Tension of Transformer Oil using UVVis Spectroscopy," Dielectrics and Electrical Insulation, IEEE Transactions on, vol. 22, pp. 1275-1282, 2015.

[29]C. Gershenson, "Artificial neural networks for beginners," arXiv preprint cs/0308031, 2003.

[30]R. A. Ghunem, K. Assaleh, and A. H. El-Hag, "Artificial neural networks with stepwise regression for predicting transformer oil furan content," Dielectrics and Electrical Insulation, IEEE Transactions on, vol. 19, pp. 414-420, 2012.

[31]V. Miranda and A. R. G. Castro, "Improving the IEC table for transformer failure diagnosis with knowledge extraction from neural networks," Power Delivery, IEEE Transactions on, vol. 20, pp. 25092516, 2005.

[32]K. F. Thang, R. K. Aggarwal, D. G. Esp, and A. J. McGrail, "Statistical and neural network analysis of dissolved gases in power transformers," in Dielectric Materials, Measurements and Applications, 2000. Eighth International Conference on (IEE Conf. Publ. No. 473), 2000, pp. 324329.

[33] A. Krogh and J. Vedelsby, Neural network ensembles, cross validation, and active learning. Cambridge MA: MIT Press, 1995.

[34] T.J. Ross, Fuzzy logic with engineering applications. 3rd ed: John Wiley \& Sons, 2009.

[35]N. A. Bakar, A. Abu-Siada, S. Islam, and M. F. El-Naggar, "A novel UV-Vis spectroscopy application to measure interfacial tension of transformer oil," IEEE Power \& Energy Society General Meeting, ,Denever, USA, 13 Jul - 02 Aug, 2015.

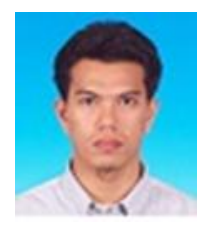

Norazhar Abu Bakar received the BEng.(Hons) degree in Electronics and Electrical from Leeds University, UK and the MSc.(Eng.) degree in Control Systems from Sheffield University, UK. He served as a lecturer at Universiti Teknikal Malaysia Melaka, Malaysia. Currently he is a $\mathrm{PhD}$ student at Curtin University. His research interests are in condition monitoring and advanced control systems.

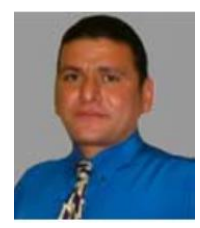

A. Abu-Siada (M'07, SM'12) received his B.Sc. and M.Sc. degrees from Ain Shams University, Egypt and his $\mathrm{PhD}$ degree from Curtin University, Australia, all in Electrical Engineering. Currently he is a Senior Lecturer in the Department of Electrical and Computer Engineering at Curtin University. His research interests include power system stability, condition monitoring, power electronics and power quality. He is Editor-in-Chief of the international journal Electrical and Electronic Engineering, a regular reviewer for IEEE Transactions on Dielectrics and Electrical Insulation, and Power Electronics and Sustainable Energy. He is vice-chair of the IEEE Computation Intellegence Society, WA Chapter. 